\title{
Metric in Reference System Transformation
}

\author{
Lu Shan 1
}

\author{
${ }^{1}$ Zhuhai Entry-Exit Inspection and Quarantine Bureau, Zhuhai, China
}

\begin{abstract}
In the past physics the assumption in the Principle of Relativity that the metrics for the reference systems are completely equivalent always exists, i.e. the statement of metric in reference system transformation had an absolute significance, which are irrelevant to the motion state of the reference systems. Through the experiment of the wave motion on the water surface caused by translating the uniform falling water drops the author finds that the wave motion velocity is mutually independent and constant energy release velocity, not related to the motion state of the uniform falling water drops parallel to the water surface. If the uniform falling water drops are regarded as wave sources or clocks, the wavelengths or periods of relatively moving wave sources or clocks differ for different motion velocities and directions. Here we show that the metrics for the relatively moving reference systems vary in different motion states if the wave sources or clocks are regarded as reference systems, with each reference system having its own metric. The metrics in reference system are of no practical significance unless the metrics for the reference system are specified. Therefore, the universality of the assumption in the Principle of Relativity that the metrics for the reference systems are completely equivalent is insufficient for describing the natural phenomena. The conflict between the Principle of Light Propagation and the Principle of Relativity will disappear only if the assumption is abandoned.
\end{abstract}

Keywords: Reference System; Metric; Wave Motion; Time

PACS: 03.30.+p; 06.30.Ft; 42.25.Bs; 06.20.F-

\section{Introduction}

In the past physics the assumption in the Principle of Relativity that the metrics for the reference systems are completely equivalent always exists, i.e. the statement of metric in reference system transformation has an absolute significance, which are irrelevant to the motion state of the reference systems. However, at the end of the $19^{\text {th }}$ century, James Clerk Maxwell proposed the electromagnetic theory based on the previous research works. According to him, the electromagnetic wave (light) velocity in vacuum is a constant, while the wave velocities of light sources must be different in the relatively uniform motion reference systems according to Galileo Galilei's velocity superposition principle $^{[1-6]}$. Since the light source moves very quickly, the wave motion velocity is beyond human's observable scale, which makes it even harder to discuss the whole process of the wave motion change in relatively moving reference systems. Therefore, it helps solve the problem to find an experiment method to get such wave motion velocity within an observable scale. As both mechanical wave and electromagnetic wave have all characteristics of wave during propagation, and the wave velocity on the water surface caused by uniform falling water drops is within the observable scale, the experiment of the wave motion on the water surface caused by translating the uniform falling water drops could provide the new thinking for the universality of the assumption in the Principle of Relativity that the metrics for the reference systems are completely equivalent.

\section{Experimental method and results}

\subsection{Principle}

The experiment was mainly composed of a translatable drip meter and a $25 \times 50 m$ pond. The basic design principle is shown in Fig. 1: A drip meter was moved linearly at a uniform velocity parallel to the water surface. As the wave motion velocity on the water surface caused by uniform falling water drops was within the observable scale, the unequivalence of metrics in transformation for different reference systems could be demonstrated by comparing the interval change of the wave motion

This article is published under the terms of the Creative Commons Attribution License 4.0

Author(s) retain the copyright of this article. Publication rights with Alkhaer Publications.

Published at: http://www.ijsciences.com/pub/issue/2016-04/

DOI: 10.18483/ijSci.995; Online ISSN: 2305-3925; Print ISSN: 2410-4477 
generated on the water surface caused by the uniform falling water drops in stationary state and uniform linear motion state parallel to the water surface.

\subsection{Apparatus and Materials}

A $25 \times 50 m$ pond above which a linear track with adjustable height was installed, which was in parallel to the water surface. A drip meter adjustable to uniform motion velocity, a digital video ( $D V)$ camera, and a measuring rule.

\subsection{Method and Steps}

The method and steps are as follows: Keep the drip meter stationary first, adjust the height of the track to observe the wave motion velocity on the water surface; select the wave motion interval (i.e., wavelength) on the water surface by adjusting the water drop rhythm of the drip meter after selecting the wave motion velocity on the water surface at a certain height. With the height and rhythm of the falling drops from the drip meter being selected, let the drip meter carry out uniform linear motion along the track parallel to water surface at different velocities $u($ as $0<u<v, u=0, u<v, u>v)$. Use a digital video $(D V)$ camera to record the wave motion status on the water surface generated by the water drops, and then use an image capture application to convert the video into divided action pictures at the time point when the water drops touch the water surface; select the pictures at the same time point when the water drops touch the water surface with the drip meter at different velocities of uniform linear motion respectively to conduct analysis and measurement, and compare the results of the velocities and compare the the results of wavelengths of wave motion on the water surface caused by the uniform falling water drops between stationary state and uniform linear motion state of the drip meter.

\subsection{Results}

Under given conditions (e.g., falling water drops had uniform rhythm, unchanged height, neglected energy consumption, etc.; the same below), the velocity of wave motion $v$ on the water surface generated by falling water drops with uniform linear translation is unchanged constantly, not related to the uniform linear motion velocity $u$ of the falling water drops with uniform linear translation.

When $u=0$, there is a set of wave surfaces formed with wave faces isotropic. See Fig. $1 a$. The wave faces generated late are always located inside and those generated early are always located outside, and the wavelength is $\lambda_{x^{\prime}}^{\prime+}=\lambda_{x^{\prime}}^{\prime-}=\lambda$.

When $0<u<v$, if $u=v / 2$, there is a set of wave surfaces formed on the water surface, with wave faces along the moving direction of water drops and wave fronts accumulated. See Fig. $1 b$. The wave faces generated late are always located inside and the wave fronts generated early are always located outside, and the wavelength along the moving direction of water drops is $\lambda_{x^{\prime}}^{\prime+}=\lambda / 2$, and that away from the moving direction of falling water drops is $\lambda_{x^{\prime}}^{\prime-}=3 \lambda / 2$.

When $u=v$, there is a set of wave surfaces formed on the water surface, with wave faces along the moving direction of water drops and wave fronts superposed. See Fig. $1 c$. The wavelength along the moving direction of water drops is $\lambda_{x^{\prime}}^{\prime+}=0$, and that away from the moving direction of falling water drops is $\lambda_{x^{\prime}}^{\prime-}=2 \lambda$.

When $u>v$, if $u=3 v / 2$, there is a set of wave surfaces formed on the water surface, with wave faces along the moving direction of water drops and wave fronts impacted. See Fig. $1 d$. The wave faces generated late rush out of the wave faces generated early; the wave faces along the moving direction of water drops are reversed, with wavelength of $\lambda_{x^{\prime}}^{\prime+}=\lambda / 2$, and the wavelength away from the moving direction of falling water drops is $\lambda_{x^{\prime}}^{\prime}=5 \lambda / 2$.

\section{Calculation and Analysis}

Under given conditions, the potential energy of the water drops is set as $E_{h}=m g h_{v}$, and the kinetic energy of the falling water drops is $E_{v}=m v^{2} / 2$; then according to the principle of conservation of energy, the following can be obtained from $E_{h}=E_{v}$ $m g h=m v^{2} / 2$

Therefore, the wave motion velocity on the water surface generated by uniform falling water drops is $v=\sqrt{2 g h}$

Where, $g$ is the gravitational acceleration. As $h$ doesn't change, $v$ is characteristic constant.

Introducing the concept of 3D Cartesian coordinate system to substitute for the reference system, the water surface is reference system $k$, expressed with $x, y, z$; the falling water drops are reference system $k^{\prime}$, expressed with $x^{\prime}, y^{\prime}, z^{\prime}$; for the sake of analysis, the screenshot which the water drop touches with the water surface being obtained, $z$ and $z^{\prime}$ in the coordinate may not be taken into consideration, as shown in Fig. 2; the wave motion velocity on the water surface generated by falling water drops is set as $v$, the falling water drop rhythm is set as $n$, when $k^{\prime}$ is stationary relative to $k$, the wavelength is $\lambda$; when $k^{\prime}$ is in uniform linear motion state at 
velocity $u$ relative to $k$, let $x^{\prime}$ axis coincide with $x$ axis permanently; the ratio of $u$ to $v$ is $m=u / v$, taking the time point when $k, k^{\prime}$ coincide with each other as the starting point for calculation.

Therefore, in the initial state when the first water drop falls onto the water surface, $k, k^{\prime}$ coincide with each other, as shown in Fig. $2 a, k^{\prime}$ moves a distance $x_{m}=0$ relative to $k$ along the direction of $x$ axis; at the same time, the wave motion on the water surface caused by the water drop is ready to diffuse outwards away from $o$ at a velocity of $v$ with such point above as the circle center, with wave motion radius of $r_{0}=0$.

In the initial state when the second water drop falls onto the water surface, as shown in Fig. $2 b, k^{\prime}$ moves a distance $x_{m}=m(\lambda)$ relative to $k$ along the direction of $x$ axis; meanwhile, the wave motion on water surface caused by the water drop is ready to diffuse outwards away from the point whose distance relative to $o$ is $x_{m}=m(\lambda)$ at a velocity of $v$, with such point as the circle center, wave motion radius of $r_{0}=0$; whereas the wave motion on the water surface caused by the first water drop diffuses outwards a unit distance $\lambda$ away from $O$ at a velocity of $v$ with such point above as the circle center, forming a wave front with such point above as the circle center and wave motion radius of $r_{1}=\lambda$.

In the initial state when the third water drop falls onto the water surface, as shown in Fig. $2 c, k^{\prime}$ moves a distance $x_{m}=m(2 \lambda)$ relative to $k$ along the direction of $x$ axis; meanwhile, the wave motion on the water surface caused by the water drop is ready to diffuse outwards away from the point whose distance relative to $o$ is $x_{m}=m(2 \lambda)$ at a velocity of $v$ with such point above as the circle center, wave motion radius of $r_{0}=0$; whereas the wave motion on water surface caused by the second water drop continuously diffuses outwards a unit distance $\lambda$ away from the point whose distance relative to $O$ is $x_{m}=m(\lambda)$ at a velocity of $v$ with such point above as the circle center, forming a wave face with such point above as the circle center and wave motion radius of $r_{1}=\lambda$; and the wave motion on the water surface caused by the first water drop continuously diffuses outwards a unit distance $\lambda$ away from $o$ at a velocity of $v$ with such point above as the circle center, forming a wave front with such point above as the circle center and wave motion radius of $r_{2}=2 \lambda$.

In the initial state when the fourth water drop falls onto the water surface, as shown in Fig. $2 d, k^{\prime}$ moves a distance $x_{m}=m(3 \lambda)$ relative to $k$ along the direction of $x$ axis; meanwhile, the wave motion on the water surface caused by the water drop is ready to diffuse outwards away from the point whose distance relative to $o$ is $x_{m}=m(3 \lambda)$ at a velocity of $v$ with such point above as the circle center, and wave motion radius of $r_{0}=0$; whereas the wave motion on water surface caused by the third water drop continuously diffuses outwards a unit distance $\lambda$ away from the point whose distance relative to $o$ is $x_{m}=m(2 \lambda)$, at a velocity of $v$, forming a wave face with such point above as the circle center and wave motion radius of $r_{1}=\lambda$; the wave motion on the water surface caused by the second water drop continuously diffuses outwards a unit distance $\lambda$ away from the point whose distance relative to $o$ is $x_{m}=m(\lambda)$, at a velocity of $v$, forming a wave face with such point above as the circle center and wave motion radius of $r_{2}=2 \lambda$; and the wave motion on the water surface caused by the first water drop continuously diffuses outwards a unit distance $\lambda$ away from $o$ at a velocity of $v$ with such point above as the circle center, forming a wave front with such point above as the circle center and wave motion radius of $r_{3}=3 \lambda$.

By analogy, in the initial state when the $(n+1)^{\text {th }}$ $(n=0,1,2,3 \cdots \cdots)$ water drop falls onto the water surface, $k^{\prime}$ moves a distance $x_{m}=m(n \lambda)$ relative to $k$ along the direction of $x$ axis; meanwhile, the wave motion on the water surface caused by the water drop is ready to diffuse outwards away from the point whose distance relative to $o$ is $x_{m}=m(n \lambda)$ at a velocity of $v$ with such point above as the circle center, and wave motion radius of $r_{0}=0 ; \ldots .$. the wave motion on the water surface caused by the first water drop continuously diffuses outwards a unit distance $\lambda$ away from $o$ at a velocity of $v$ with such point above as the circle center, forming a wave front with such point above as the circle center and wave motion radius of $r_{n}=n \lambda$. Therefore, the wave motions on the water surface caused by translating uniform falling water drops are a set of wave surfaces with water drop falling positions $o^{\prime}$ as centers of circles, with radius of $r_{n}=n \lambda(n=0,1,2,3 \cdots \cdots)$, respectively. 
If the uniform falling water drops are regarded as wave sources, as shown in Fig. 3, the distance that wave source $k^{\prime}$ moves relative to $k$ is

$x_{m}=m(n \lambda)=n m \lambda$

2D wave motion equation of wave source $k^{\prime}$ relative to $k$ can be obtained with mathematical description.

$\left[x^{\prime}+m r_{n}\right]^{2}+\left(y^{\prime}\right)^{2}=\left(r_{n}\right)^{2}$

Similarly, if wave sources are regarded as clocks ${ }^{[7]}$, then the time of the clocks can be introduced into the space by assuming that the clocks always generate physical reality in their surrounding space, just like wave motions generated by wave sources, according to the electromagnetic field description method by Michael Faraday ${ }^{[8]}$. Then, the wave sources are equal to clocks, the wave motion velocities of wave sources are equal to the velocities of clocks, the wave motion distances are equal to the time lengths of clocks, the wavelengths are equal to the time periods of clocks, and the wave motion rhythms are equal to the time rhythms. Since the wave motion velocity $v$ is always unchanged, letting $t_{n}=r_{n} / v, t_{x^{\prime}}^{\prime}=x^{\prime} / v$, $t_{y^{\prime}}^{\prime}=y^{\prime} / v$, the 2D time equation of clock $k^{\prime}$ relative to $k$ can be obtained by dividing both sides of Eq. (2) by $v^{2}$.

$\left[t_{x^{\prime}}^{\prime}+m t_{n}\right]^{2}+\left(t_{y^{\prime}}^{\prime}\right)^{2}=\left(t_{n}\right)^{2}$

If only the wave motion distance of the wave source or time length of the clock in the direction of $x^{\prime}$ axis is taken into account, the following can be gotten from Eq. (2) at $y^{\prime}=0$ and Eq. (3) at $t_{y^{\prime}}^{\prime}=0$ by letting $T=\lambda / v$ :

Along the $x^{\prime}$ axis, the wave motion distance of the wave source or the time length of the clock is

$x^{\prime+}=(1-m) n \lambda, t^{\prime+}=(1-m) n T$

Away from the $x^{\prime}$ axis, the wave motion distance of the wave source or the time length of the clock is

$x^{\prime-}=-(1+m) n \lambda, t^{\prime-}=-(1+m) n T$

The following can be gotten by letting $n+1$ minus $n$ in Eq. (4) and Eq. (5):

Along the $x^{\prime}$ axis, the wave length of the wave source or the period of the clock is

$\lambda_{x^{\prime}}^{\prime+}=(1-m) \lambda, T_{x^{\prime}}^{\prime+}=(1-m) T$

Away from the $x^{\prime}$ axis, the wave length of the wave source or the period of the clock is

$\lambda_{x^{\prime}}^{\prime-}=-(1+m) \lambda, \quad T_{x^{\prime}}^{\prime-}=-(1+m) T$

As $\lambda_{x^{\prime}}^{\prime+}-\lambda_{x^{\prime}}^{\prime-}=2 \lambda$, such phenomenon that the contraction of the wavelengths or the periods along the movement direction of the wave source or the clock and the expansion of the wavelengths or the periods against the movement direction of the wave source or the clock offset each other in amplitude is called asymmetric frequency shift.

Similarly, if only the wave motion distance of the wave source or the time length of the clock in the direction of $y^{\prime}$ axis is taken into account, then the following can be gotten from Eq. (2) at $x^{\prime}=0$ and Eq. (3) at $t_{x^{\prime}}^{\prime}=0$ by letting $T=\lambda / v$ :

$y^{\prime}= \pm n \lambda \sqrt{1-m^{2}}, \quad t_{y^{\prime}}^{\prime}= \pm n T \sqrt{1-m^{2}}$

So, the wavelength of the wave source or the period of the clock in the direction of $y^{\prime}$ axis obtained by letting $n+1$ minus $n$ is:

$\lambda_{y^{\prime}}^{\prime}= \pm \lambda \sqrt{1-m^{2}}, \quad T_{y^{\prime}}^{\prime}= \pm T \sqrt{1-m^{2}}$

Where, the direction along $y^{\prime}$ axis is taken as positive, and that away from the direction of $x^{\prime}$ axis is taken as negative.

As $\lambda_{y^{\prime}}^{\prime+}+\lambda_{y^{\prime}}^{\prime-}=0$, such phenomenon that perpendicular to the movement direction of the wave source or the clock the wavelengths or periods contract simultaneously with equal amplitudes and reverse directions is called symmetric frequency shift.

It can be seen that, as $v$ is constant when $m=0$, i.e., $u=0$, as shown in Fig. $3 a$, their wavelengths of the wave source or the periods of the clocks are equal in amplitude and isotropic. When $m>0$, i.e., $u>0$, as shown in Figs. $3 b, 3 c$ and $3 d$, their wavelengths or periods are different in different motion velocities and directions.

\section{Discussion}

Previous research efforts show that, whether the theory of electromagnetic wave (light) proposed by Maxwell or the experiment for existence of electromagnetic waves conducted by Heinrich Rudolf Hertz, a German physicist, made the conclusion that wave velocity does not depend on the motion of the wave source, and made an assertion in principle that the electromagnetic field equations are unchanged in form between reference systems in relatively uniform motion states. However, the aspects about the electromagnetic field quantity transformation equation and the covariance of electromagnetic field equations between the reference systems in relatively uniform motion states were not further discussed. To harmonize the contradictions between electromagnetics established in the $19^{\text {th }}$ century and the classical physics, believing that the Principle of Relativity and the invariance of light speed was right, Hendrik Norton Lorenz proposed a transformation 
relationship for observers to conduct quantity measurement between reference systems in relatively uniform motion states. However, there was a very important experiment in the history, namely, the Michelson-Morley Experiment in which the optical path difference should be observable when the Lorentz transformation was used for calculation. However, the Michelson-Morley Experiment got a completely negative result ${ }^{[1-6]}$, which make people can not help but query the premise of the derivation of Lorentz transformation. However, the invariance of light speed has been confirmed by many experiments. In contrast the universality of the Principle of Relativity has not been confirmed by experiments yet. Through the experiment of the wave motion on the water surface caused by translating the uniform falling water drops it can be found that, as the wave motion velocity of wave sources or the time velocity of clocks does not depend on the motion of wave sources or clocks, the metrics vary in different motion states as to the relative motion systems if the wave sources or the clocks are regarded as reference systems. Each reference object has its own specific metric. The metrics in reference system transformation are of no practical significance unless the metrics for the reference system are specified. Therefore, the universality of the assumption in the Principle of Relativity that the metrics for the reference systems are completely equivalent is insufficient for describing all the natural phenomena.

Although the wave motion on the water surface caused by uniform falling water drops originates from mechanical wave, many experiments have proved that all waves have identical nature, and both mechanical wave and electromagnetic wave have all characteristics of wave during propagation, i.e., there are same relationships among wave velocity, wavelength, frequency, and period. Just as the Doppler effect study originated from mechanical wave is widely used for electromagnetic waves. From Eq. (6) and (7), it can be seen that it can be rewritten as $u=\left(\lambda \mp \lambda_{x}^{\prime}\right) v / \lambda$ by substituting $m=u / v$, and its result form is completely consistent with the description of the Doppler effect. Consequently, all empirical facts supporting the Doppler effect support the experimental results of the wave motion on the water surface caused by translating uniform falling water drops.

The above description of the experimental results of the wave motion on the water surface caused by translating uniform falling water drops is only effective for some reference systems in the relatively stationary or uniform linear motion state, while such insufficient description can offer a clear basic concept for the space and time in relative motion state without which the electrodynamics of moving bodies could not be established accurately.

Obviously, as is seen from the experimental results of the wave motion on the water surface caused by translating uniform falling water drops that if wave sources or clocks are regarded as reference systems, the metrics for the reference systems are not completely equivalent. Thus, the assumption in the Principle of Relativity that the metrics for the reference systems are completely equivalent is hard to accept for the wave sources or the clocks with relative motion. Hence rethinking the Principle of Relativity could not only help unify the Galileo transformation and Lorentz transformation and obtain the strict representation (appendix) but also find that the classical physics and electromagnetics are covariant. What is more important is that the change of the metrics for the reference systems in the Principle of Relativity is based on empirical study instead of stipulation. It is the nature that the change of metrics for the reference systems in the Principle of Relativity is not completely equivalent that makes people feel the natural world alienated from the Principle of Relativity. Unfortunately physical theories do not attach great importance to this. The development of physical theories indicates that any auxiliary assumption introduced into theory should conform to natural facts. The conclusion of this research makes it worth rethinking everything ever done with the Principle of Relativity in physics theory in the past. Otherwise, all physical statements related to the Principle of Relativity will be confronted with the similar difficulty.

Therefore, discussing the Principle of Relativity with the wave motion experiment on the water surface caused by translating uniform falling water drops may provide insights into emancipation of conventional thinking.

\section{Conclusion}

The wave motion velocity of wave sources or the time velocity of clocks does not depend on the motion of wave sources or clocks. If wave sources or clocks are regarded as reference systems, the metrics vary in different motion states of relatively moving reference systems. Each reference system has its own specific metric; the metrics for relatively moving reference systems are of no practical significance unless the metrics for the reference system are specified. Therefore, the universality of the assumption in the Principle of Relativity that the metrics for the reference systems are completely equivalent is insufficient for describing the natural phenomena. The conflict between the Principle of Light Propagation and the Principle of Relativity 
would disappear only if the assumption is abandoned.

\section{Appendix}

\section{The Strict Representation of Coordinate System Transformation}

Introducing the concept of 3D coordinate system to substitute for the reference system, the orientation of the two coordinate systems $k, k^{\prime}$ is relative as shown in Fig.4, the $x, x^{\prime}$ axes of these two coordinate systems are coincident forever. We only take events happened on the $x, x^{\prime}$ axis into consideration for the sake of simplification. It can be imagined that the observers carry the clocks of the same structure respectively in the relatively moving reference systems, then any event relative to $k$ is described by the abscissa $x$ and time $t$ and the one relative to $k^{\prime}$ is described by the abscissa $x^{\prime}$ and time $t^{\prime} . k^{\prime}$ is set to move along the $x^{\prime}$ axis with the velocity of $u$ relative to $k$. The object $p$ is set to move along the $x^{\prime}$ axis with the velocity of $v_{p}$ relative to $k^{\prime}$.The time velocity of the clock is $v$ with the wave length $\lambda$, then the inherent period of the clock is $T=\lambda / v$. According to the article "Metric in Reference System Transformation", the clock period of the motion of $k^{\prime}$ along $x^{\prime}$ axis can be gotten by Eq. (6)

$T^{\prime}=(1-m) T$

Where, $m$ is the ratio between $u$ and $v$. Since $t^{\prime}=n T^{\prime}, t=n T$, the time of $p$ relative to $k^{\prime}$ is:

$t^{\prime}=(1-m) t$

The following can be obtained by substituting $m=u / v$ into (1.1)

$t^{\prime}=(1-u / v) t$

Since the moving distance of $p$ relative to $k^{\prime}$ is

$x^{\prime}=v_{p} t^{\prime}$

The following can be obtained by substituting (1.2) into (1.3)

$x^{\prime}=v_{p} t^{\prime}=v_{p}(1-u / v) t$

Meanwhile, as $k^{\prime}$ moves a distance $u t$ relative to $k$, the moving distance of the object $p$ relative to $k$ is

$x=x^{\prime}+u t$

The following can be obtained by substituting

$x=v_{p}(1-u / v) t+u t$

Further, it can be

$x=\left[v_{p}+\left(1-v_{p} / v\right) u\right] t$

It can be seen from the Eq. (1.5) that the relation of the coordinate system transformation is related to the ratio between the object motion velocity $v_{p}$ and the time velocity of the clock $v$ if the observers of the coordinate system carry the clocks of the same structure respectively. The higher the clock time velocity, the less error of the coordinate system transformation and vise versa. If the time velocity of the clock remains unchanged, the lower the object motion velocity, the less error of the coordinate system transformation and vise versa. When the object motion velocity $v_{p}$ is far less than the time velocity of the clock $v$, i.e. when $v_{p}<<c$ or $v_{p} / c<<1$, the object motion velocity relative to another coordinate system is approximate to the superposition of the object motion velocity and the motion velocity between coordinate systems, i.e. $x \approx\left(v_{p}+u\right) t$. When the object motion velocity $v_{p}$ equals to the time velocity of the clock $v$, i.e. when $v_{p}=v=c$ or $v_{p} / v=1$, the object motion velocity relative to another coordinate system is not related to the motion velocity between coordinate systems, i.e. $x=v_{p} t$.

In the real observation, since the observer's observations are all from the light, the time velocity of the observer's clock is therefore related to the light velocity. Therefore, the time velocity of the observer's clock equals to the light velocity $\left(3 \times 10^{-8} \mathrm{~m} / \mathrm{s}\right)$, i.e. $v=c$. The Galilean transformation in physics describes the object motion along the motion direction of the observer $k^{\prime}$. Such object motion velocity is far less than the time velocity of the observer's clock, i.e. $v_{p}<<c$ or $v_{p} / c<<1$. Hence, what the observer $k$ sees is the approximate superposition result of the object motion velocity relative to $k^{\prime}$ and the motion velocity of $k^{\prime}$ relative to $k$, namely $x \approx\left(v_{p}+u\right) t$. In fact, the light velocity remains constant and the object motion velocity isn't equal to zero, namely $v_{p} \neq 0$, thus the lower the object motion velocity, the less error of the Galilean transformation and vise versa. Therefore, the Galilean transformation result is in fact an approximate value. While what the Lorentz transformation describes is the light wave motion along the motion direction of the observer $k^{\prime[2-4]}$. Since the light wave motion velocity equals to the time velocity of the observer's clock, i.e. $v_{p}=v=c$ or $v_{p} / v=1$, what the observer $k$ sees along the motion direction of the observer $k^{\prime}$ is the result that light velocity is constant, i.e. $x=c t$.

Normally, for the sake of the factual connection of the clock and nature, the conventional physical clock is set on the earth according to the sun. The time 
velocity of such clock is $7.292 \times 10^{-5}$ radians per second which is $465 \mathrm{~m} / \mathrm{s}$ on the earth's equator, i.e. $v=465 \mathrm{~m} / \mathrm{s}$. Since the object motion velocity within people's visual observable scale is less than the sound velocity (the sound velocity in the air is $346 \mathrm{~m} / \mathrm{s}$ ), more velocity superposition cases are manifested in the normal observation of objects in low velocity motion. Galileo velocity superposition principle will be much less affected if the observer's time velocity of the clock equals to the light velocity which is far greater than the object motion velocity. As Lorentz transformation is derived by the Principle of Relativity based on the Galilean transformation which is actually an approximate value, the derived result in turn is inevitably an approximate value which can be clearly seen in the Michael-Morley Experiment. The optical path difference can be observed either in Galileo's velocity superposition principle or in Lorentz transformation calculation. What is different is that the result from Lorentz transformation calculation is less than that from Galileo's velocity superposition principle ${ }^{[5-6]}$. However, the Michael-Morley Experiment got a completely negative result. However, calculated from Eq. (1.5), when $v_{p}=v=c$ or $v_{p} / v=1$, the result is consistent with the result of the Michael-Morley

\section{Experiment.}

Hence, the time velocity of the clock does not depend on the clock motion according to the result of "Metric in Reference System Transformation". As to the clock in the state of relative motion, the period varies in different motion states if the clock is regarded as the reference system. It can be found that the observer determines the location of the reference system relative to himself according to the time described by each "tick" of the clock he takes. Thus, the relative time velocity is involved in the issue of place and time of any motion direction. What is more important is that the Galileo transformation and Lorentz transformation could be unified and obtain a strict representation, (i.e. Eq. 1.5); such result not only applies to the low velocity motion of the classical physics but also to the high velocity motion of electromagnetics. Thus the conflict between the classical physics and the electromagnetics will disappear. It's not difficult to find that it is the time change described by each clock "tick" that makes it worthwhile for the physics theories in the past to rethink all the things introduced by the conception of time in the relative motion. 

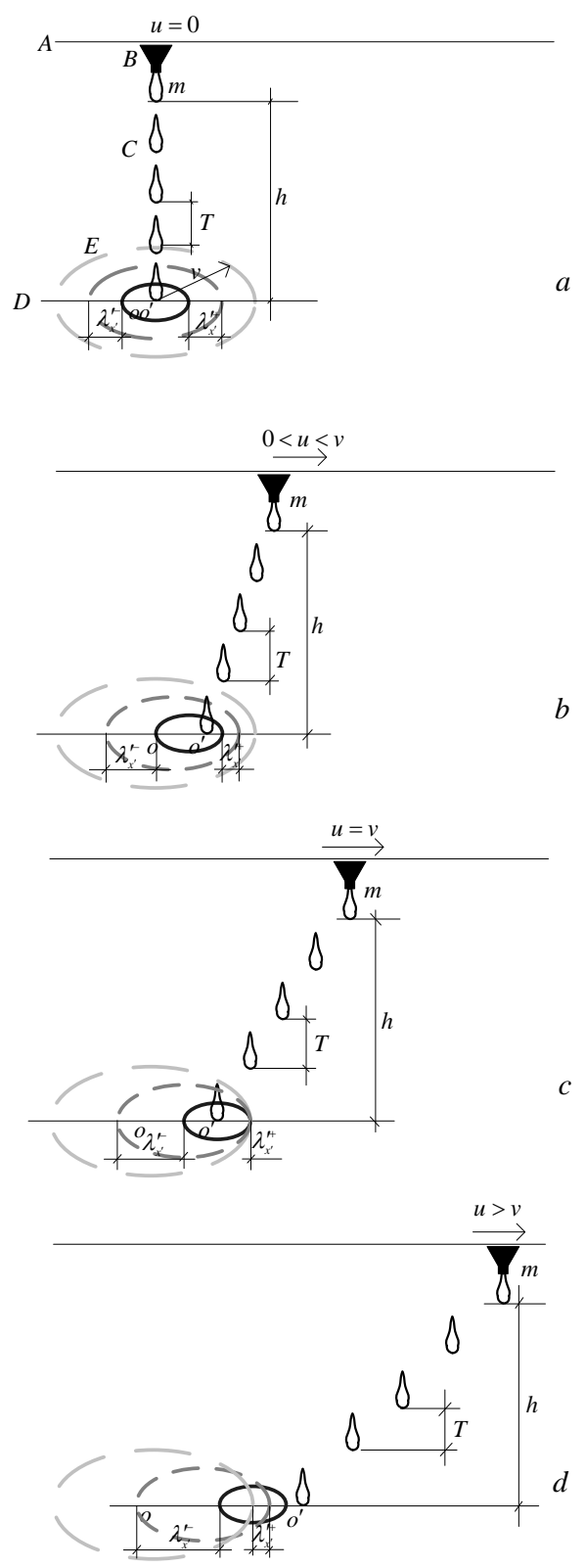

A is track, B is dropper, C is water drop, $\mathrm{D}$ is water surface, and $\mathrm{E}$ is water wave

Fig. 1 Experiment of wave motion on the water surface caused by translating uniform falling water drops
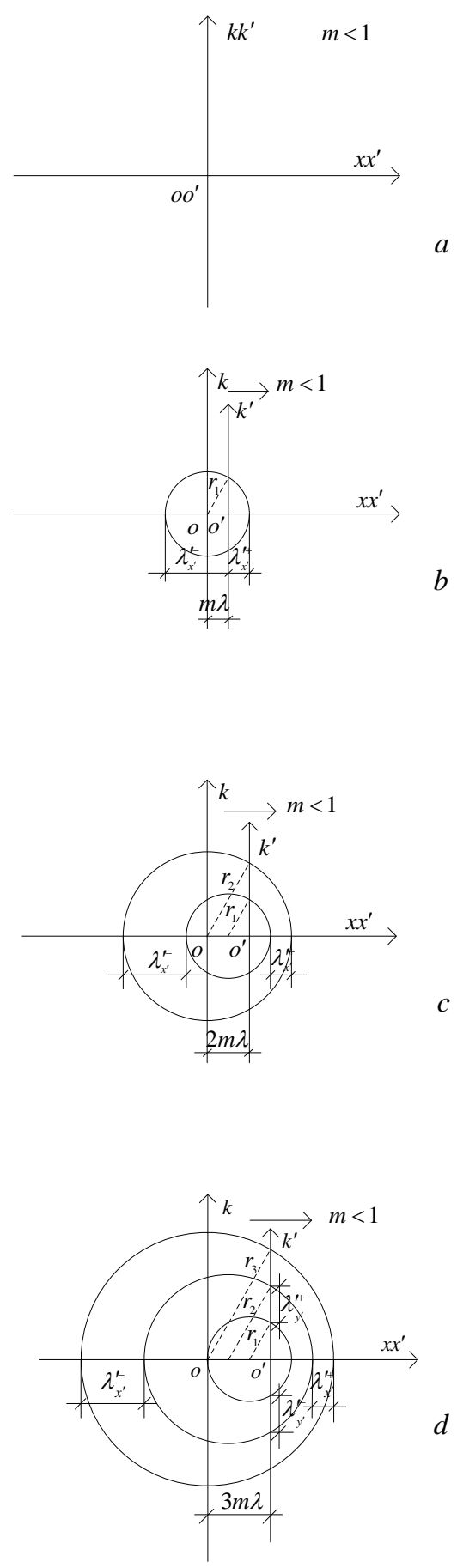

Fig. 2 Process of wave motion on the water surface caused by translating uniform falling water drops 


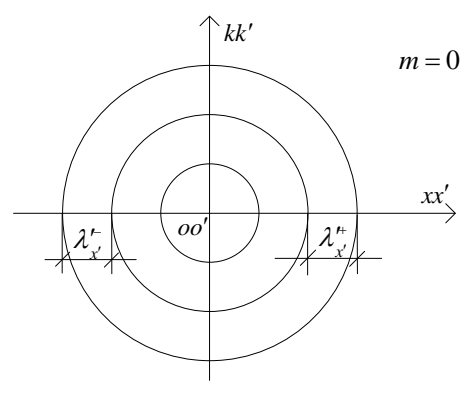

$a$
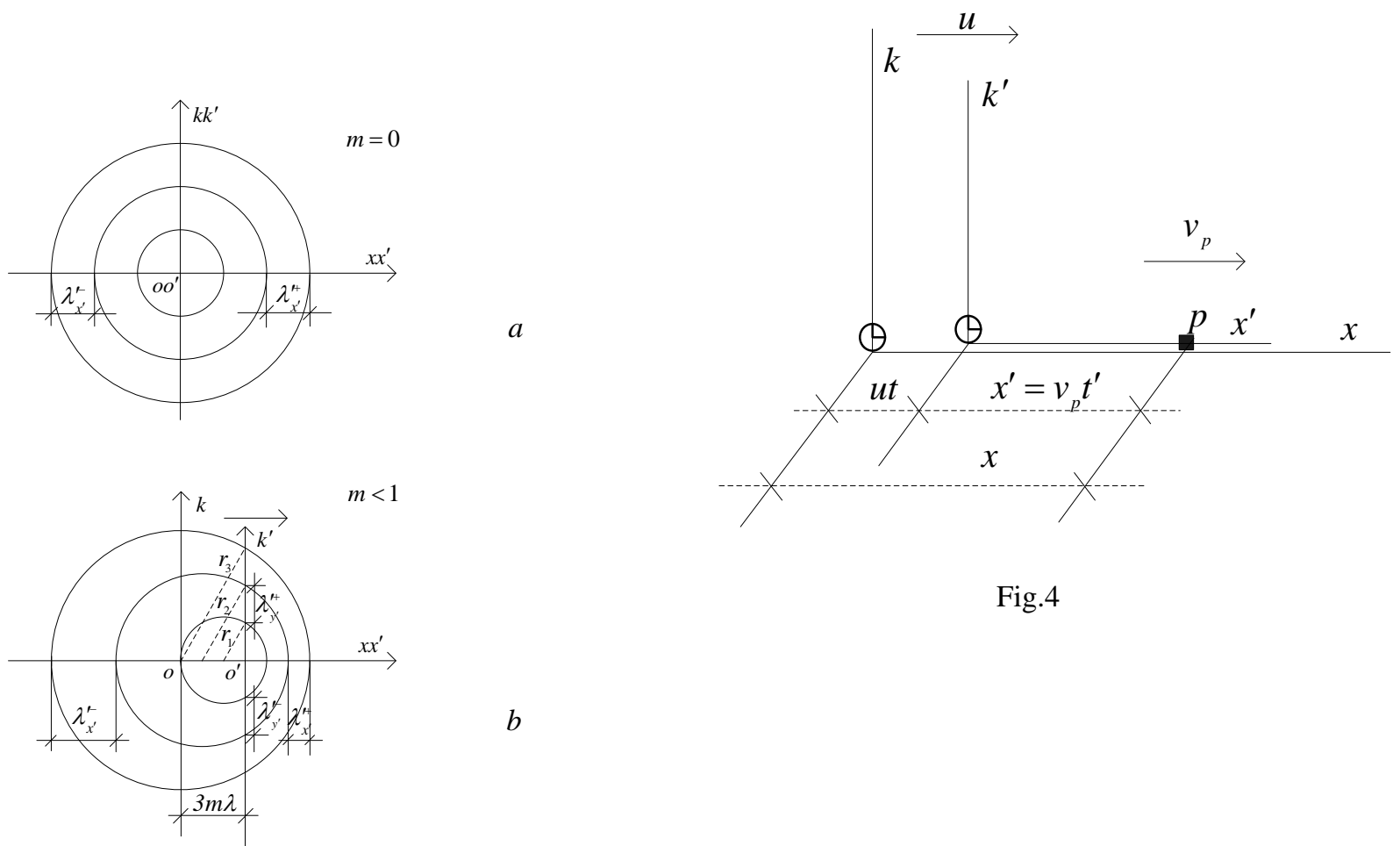

Fig.4

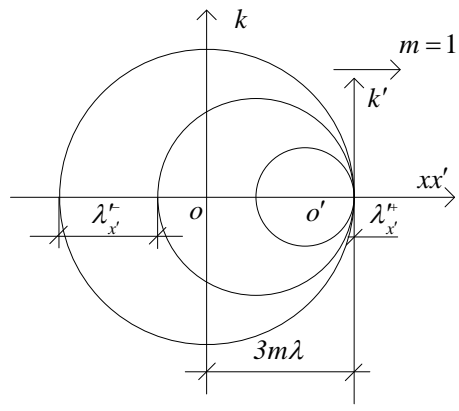

c

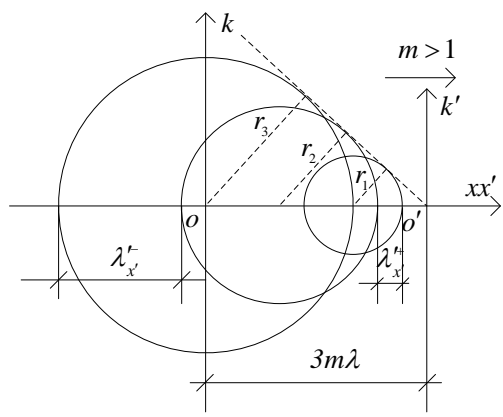

$b$

$d$

Fig. 3 Results of wave motion on the water surface caused by translating uniform falling water drops 


\section{Acknowledgement:}

I would like to express my deepest gratitude to the Associate Professor Liu Haiping for her translation, as this thesis would come out quite late without her sincere help. My gratitude also goes to my colleagues Jin Ning, Gao Yansheng, Mo Jianbin and Wang Yiduo for their suggestions and providing convenience during submitting.

\section{References}

1) Henri Poincare (France). Translated into Chinese by $\mathrm{Li}$ Xingmin. La Science et l'Hypothèse (Science and Hypothesis). (The Commercial Press, Beijing ,August 2006, 65-75, 103-130,189-190)

2) Henri Poincare (France). Translated into Chinese by $\mathrm{Li}$ Xingmin. Science et Méthode (Science and Method), (The Commercial Press, Beijing, December 2006, 69-86, 165-185,
175)

3) Xiang Yihe. Source Tracing for Fundamental Concepts and Fundamental Laws in Physics. (Higher Education Press, Beijing , September 1994, 120-199, 219-255)

4) Maxwell, on Farday's lines of Force, ed. W. D. Niven, The Scientific papers of James clerk Maxwell, Cambridge University Press, Vo1.1, (1890) pp155-209

5) Tetu Hirosige (Japan). Translated into Chinese by $\mathrm{Li}$ Xingmin. Historical Studies in the Physical Sciences. (Qiushi Publishing House, Beijing, May 1988, 293-305, 602-604, 626-631, 637-640,

6) A. Einstein, et al. Translated into Chinese by Zhao Zhitian and Liu Yiguan. The Principle of Relativity (Classic papers about special relativity and general relativity). (Science Press, Beijing , February 1980, 1-5, 31-54)

7) A. $\cdot$ G. Smith. Translated into Chinese by Zhang Yanhua. The Origin of Time. (Shandong Pictorial Publishing House, Shandong, May 2006, 50-56)

8) Faraday, Experimental Researches in Electricity, VOL. II, London, I . M. Dent and sons, LTD, 159-162 